\title{
Development of PPD Sensitive to Deep UV Scintillation Photons of Liquid Xenon
}

\author{
W. Ootani*, X. Bai, T. Chiba, Y. Fujii, T. Iwamoto, D. Kaneko, T. Mori, H. Natori, \\ R. Sawada, Y. Uchiyama \\ ICEPP, University of Tokyo, 7-3-1 Hongo, Bunkyo-ku, Tokyo 113-0033, Japan \\ E-mail: wataru@icepp.s.u-tokyo.ac.jp \\ S. Mihara, H. Nishiguchi \\ KEK, High Energy Accelerator Research Organization, 1-1 Oho, Tsukuba, Ibaraki 305-0801, \\ Japan
}

\section{A. Stoykov}

Paul Scherrer Institut, CH-5232 Villigen, Switzerland

\begin{abstract}
We are developing Pixelated Photon Detector (PPD) sensitive to deep UV scintillation photons of liquid xenon as a possible candidate for the photosensor upgrade of the MEG liquid xenon $\gamma$-ray detector. The prototypes of the PPD preliminarily optimized for the detection of deep UV photons were tested in LXe and their basic properties were measured. The measured performance of the prototypes is described.
\end{abstract}

International Workshop on New Photon-detectors,

June 13-15, 2012

LAL Orsay, France

${ }^{*}$ Speaker. 


\section{Introduction}

Liquid xenon ( $\mathrm{LXe})$ is known to have excellent properties as a detector medium and is currently used or planned to be used for various projects in particle and nuclear physics or medical sciences. One example is the MEG experiment searching for the lepton flavor violating decay of muon, $\mu^{+} \rightarrow e^{+} \gamma$, where a $900 \ell$ LXe scintillation detector is employed to detect $52.8 \mathrm{MeV} \gamma$-rays from the $\mu^{+} \rightarrow e^{+} \gamma$ decay and the LXe scintillation light in deep UV range ( $\lambda=178 \pm 14 \mathrm{~nm}$ [1]) is currently detected by 2 -inch UV-sensitive photomultiplier tubes (PMTs) [2]. The energy and position resolutions of the current detector are, however, limited due to the non-uniform PMT coverage. We plan to upgrade the LXe detector by replacing the current 2-inch PMTs with smaller photosensors to improve the resolutions with more uniform and highly granular scintillation readout. Pixelated Photon Detector (PPD) [3], which is a new type of semiconductor-based photosensor also known as SiPM, MPPC, G-APD, etc., is a good candidate for the replacement of the PMT. We are developing a large-area PPD sensitive to LXe scintillation in collaboration with Hamamatsu Photonics. The performance of the prototypes which is preliminary optimized for the detection of deep UV photons is presented.

\section{Development of UV-enhanced PPD}

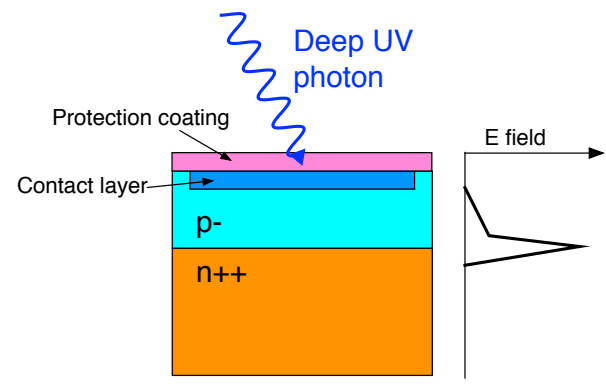

Figure 1: Typical layer structure of a commercial PPD.

Major requirements for the PPD for the MEG LXe detector are (1) sensor area of about $10 \times$ $10 \mathrm{~mm}^{2}$, (2) Photon Detection Efficiency (PDE) for the LXe scintillation light $>10 \%$, (3) gain $>10^{6}$. The most important parameter is the PDE since commercial PPDs are not sensitive to deep UV light. The low sensitivity to the deep UV light results from the fact that the deep UV photons are mostly absorbed in the protection coating, usually made of epoxy resin, and in the thin contact layer with no strong electric field, or are reflected on the silicon surface before reaching the sensitive layer, where the avalanche amplification occurs as illustrated in Fig 1. Possible solutions would be to remove the protection coating, to make the contact layer as thin as possible and to minimize the reflection by optimizing the optical coating on the sensor surface. The PDE, gain and signal width have to be balanced by adjusting the quench resistor and pixel size.

\section{Properties of Prototypes at LXe Temperature}

We tested different prototypes (labeled as A to H) of the UV-enhanced PPD, produced by 
Hamamatsu Photonics, in a LXe test facility at the Paul Scherrer Institute (PSI) in Switzerland. The sensor area is $3 \times 3 \mathrm{~mm}^{2}$ and the pixel pitch is $50 \mu \mathrm{m}$ or $100 \mu \mathrm{m}$. There is no protection coating for all the types. For the types A to F, the contact layer is thinner than for the commercial one and the anti-reflection coating is optimized, while for the type $\mathrm{G}$ and $\mathrm{H}$, the refractive index on the sensor surface is optimized for LXe.

The prototypes are mounted in a small cryostat filled with $2 \ell$ LXe as illustrated in Fig. 2. An $\alpha$-source (Am-241) is installed for the absolute measurement of the PDE. Note that the $\alpha$-event can be regarded as a point-like and monochromatic scintillation light source. The expected number of incoming scintillation photons on the sensor area of $3 \times 3 \mathrm{~mm}^{2}$ is $O(100)$. An UV-sensitive PMT is also installed for triggering on the $\alpha$-event. The whole setup is surrounded by a cylindrical wall with a special coating to suppress the reflection of the UV-photons. The signal from the PPD is amplified by a voltage amplifier (gain of 10 , bandwidth of $1 \mathrm{GHz}$ ) placed outside the cryostat and then readout by a waveform digitizer ${ }^{1}$.

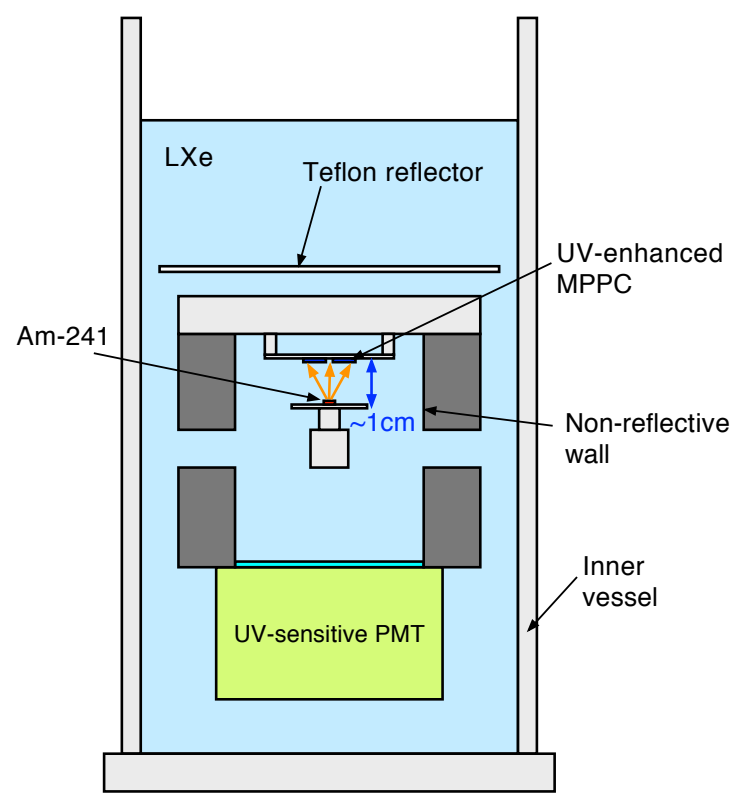

Figure 2: Schematic view of the setup of the prototype test. MPPCs under test are illuminated by direct scintillation light from $\alpha$-source (Am-241). The PMT signal generated by the light reflected on a Teflon plate on top is used for triggering on the $\alpha$-event.

The LXe scintillation is successfully detected by the prototypes. Fig. 3 shows a typical signal from the prototype (type G) for the $\alpha$-event where the PMT signal for the same event is also shown. The longer tail of the PPD signal is due to the long tail of the single photoelectron signal of the PPD, which is measured to be $40 \mathrm{~ns}$. The long tail of the single photoelectron signal is caused by the increased resistivity of the quench resistor made of poly-silicon at the LXe temperature of $165 \mathrm{~K}$. The resistance of the quench resistor is measured by applying a forward voltage at both room and LXe temperatures. The quench resistor typically shows a few times higher resistivity at

\footnotetext{
${ }^{1}$ Both the amplifier and waveform digitizer (DRS4) [4] are in-house developed at PSI.
} 
LXe temperature compared to room temperature. For example, the resistances for the type $\mathrm{G}$ are measured to be $102 \mathrm{k} \Omega$ and $272 \mathrm{k} \Omega$ at room and LXe temperatures, respectively.

The dark count rate is found to be reduced from $1 \mathrm{MHz}$ at room temperature down to $10 \mathrm{~Hz}$ at LXe temperature at an overvoltage of $1 \mathrm{~V}$. The reduction of five orders of magnitude is consistent with the previous measurements [5] although the absolute rate is somewhat higher. The measured dark count rate at LXe temperature is low enough for the MEG LXe detector.

In contrast, the correlated noise such as the optical crosstalk and afterpulsing is expected to remain at low temperature. The probabilities of the optical crosstalk and afterpulsing are measured to be ranging from 5 to $20 \%$ and from 10 to $30 \%$, respectively depending on the types and the overvoltage.
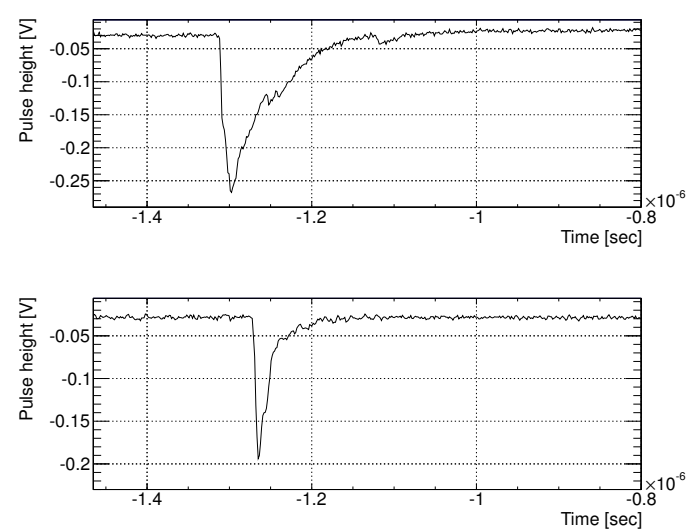

Figure 3: Typical signals from the PPD prototype (upper) and PMT (lower) for the same $\alpha$-event.

The gains of the prototypes measured from the single photoelectron signal are shown on the left in Fig. 4. All the PPDs with $50 \mu \mathrm{m}$ pixel pitch show a similar gain of $10^{6}$ at an overvoltage of $1 \mathrm{~V}$, which already meets our requirement. The prototype with $100 \mu \mathrm{m}$ pixel pitch shows a higher gain due to its larger pixel capacitance as expected.

The absolute PDE is measured as the ratio of the observed number of photoelectrons to the expected number of photons impinging on the sensor area from $\alpha$-event. Only the direct photons are taken into account in the calculation of the expectation since the attenuation of the scintillation photons in LXe should be small for the short distance of about $10 \mathrm{~mm}$ between the $\alpha$-source and the prototype and the effect of the reflection should also be small because of the non-reflecting wall. The effective PDEs are obtained as shown on the right in Fig. 4 by subtracting the effect of the correlated noise by using the measured probabilities mentioned above. The PDEs only for the type $\mathrm{F}(100 \mu \mathrm{m}$ pixel pitch), $\mathrm{G}$ and $\mathrm{H}$ are shown in the figure while the PDEs for the other types are much lower. The type $\mathrm{G}$ samples where the refractive index of the sensor surface is better matched to that of LXe show the best PDE of about $10 \%$ at an overvoltage of $1 \mathrm{~V}$. The best PDE is still lower than the quantum efficiency of about $15 \%$ of the PMT used in the current MEG LXe detector. The total collection efficiency for the LXe scintillation light in the planned new detector with PPDs with the PDE at this level is, however, expected to be comparable or even better than that of the current detector because of the higher area coverage. Therefore, the PDE of the prototype for the 
LXe scintillation photons already meets our requirement although the effect of the relatively high correlated noise has to be carefully investigated.
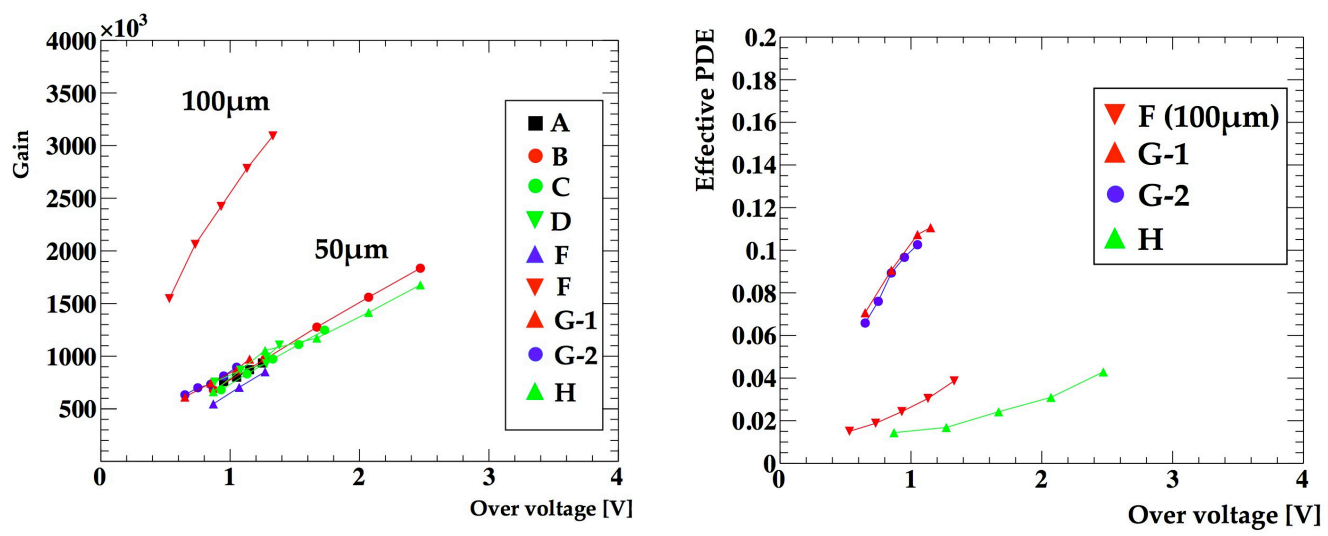

Figure 4: (Left) Measured gains of the prototypes of the UV-enhanced PPDs and (right) effective PDEs after the correction for the contributions from the optical crosstalk and afterpulsing.

\section{Test of Large Area PPD at LXe Temperature}

The size of the currently tested prototype is $3 \times 3 \mathrm{~mm}^{2}$, which is too small to fully cover the $\gamma$-ray entrance face of the MEG LXe detector of about $1 \mathrm{~m}^{2}$. It is the problem not only for the cost of the readout electronics but also for the external heat inflow to the LXe at low temperature due to the large number of cables. The size of about $12 \times 12 \mathrm{~mm}^{2}$ would, therefore, be necessary corresponding to the total number of channels of about 4000 .

The possible issues for the large area sensor would be the dark count rate, which is proportional to the area, the uniformity of the pixel gain over the whole sensor area and the effect of the large sensor capacitance. We investigated those issues by testing commercial monolithic array MPPCs (S11827-3344MG) at LXe temperature. The monolithic array MPPC is composed of $4 \times 4$ sectors fabricated on a single substrate, each of which is an independent sensor of $3 \times 3 \mathrm{~mm}^{2}$. It is equipped with 16 independent anode electrodes and a single common cathode electrode. A single sensor with an effective area of $9 \times 9 \mathrm{~mm}^{2}$ or $12 \times 12 \mathrm{~mm}^{2}$ is formed by connecting $3 \times 3$ or $4 \times 4$ sectors in parallel, respectively.

The charge spectrum for the dark noise observed in LXe with the monolithic array MPPC with $4 \times 4$ sectors connected in parallel is shown on the left in Fig. 5, where we can see that the single photoelectron peak is clearly resolved. It indicates that the pixel gain is quite uniform over the whole sensor area. This good separation is also because of the low dark count rate at LXe temperature, which is measured to be $720 \mathrm{~Hz}$ for the $4 \times 4$ connection.

There are some issues related to the larger sensor capacitance as seen in the waveforms for the single photoelectron signal shown on the right in Fig. 5. The signal fall times are measured to be about 100 and $200 \mathrm{~ns}$ for the $3 \times 3$ and $4 \times 4$ connection, respectively. The pileups caused by this longer tail could be an issue in the high rate environment of the MEG experiment. The higher noise 
for the larger sensor area seen in the waveform, which is considered as capacitive noise, could also be an issue.
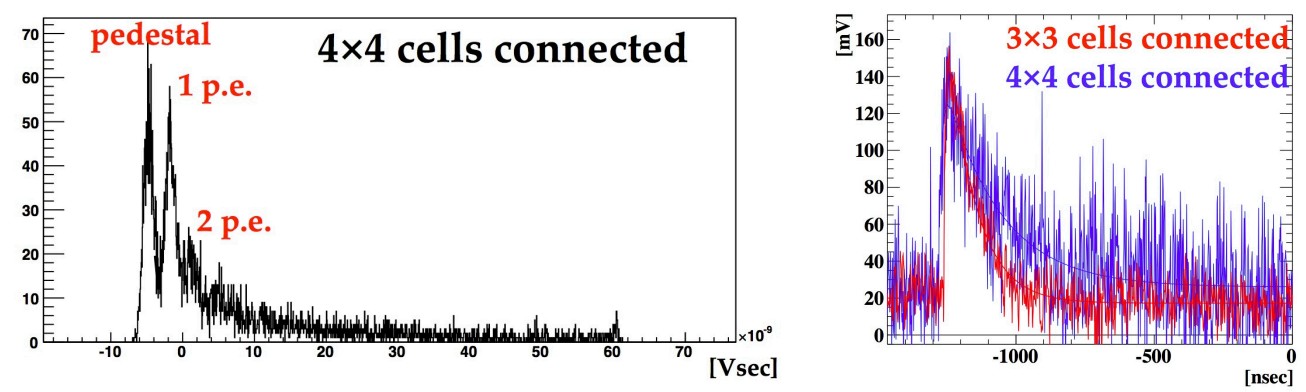

Figure 5: (Left) Charge spectrum of the dark noise for the monolithic array MPPC with all $4 \times 4$ sectors connected in parallel and (right) typical waveforms from the MPPCs with $3 \times 3$ and $4 \times 4$ sectors connected.

\section{Summary and Outlook}

UV-enhanced PPD is under development in collaboration with Hamamatsu Photonics as a possible candidate for the new photosensor for the upgrade of the MEG LXe $\gamma$-ray detector. Prototypes are tested in LXe and show promising performance such as PDE of $10 \%$, gain of $10^{6}$ and low dark count rate. The possible issues for the large area sensor are investigated using monolithic array MPPCs working as a single sensor with an effective area up to $12 \times 12 \mathrm{~mm}^{2}$ by connecting the sectors in parallel. A single photoelectron peak is clearly resolved at LXe temperature and the dark count rate is measured to be less than $1 \mathrm{kHz}$.

We are soon to test a prototype of the UV-enhanced PPD with a large area of $12 \times 12 \mathrm{~mm}^{2}$ and the production parameters of the PPD for the MEG LXe detector will be finalized. We plan to build a LXe detector prototype with $100 \ell$ LXe and 600 large area UV-enhanced PPDs and then to perform a beam test. We aim at the construction of the final detector with 4000 PPDs after proving the concept of the new detector with the prototype detector.

\section{Acknowledgement}

This work is partly supported by MEXT KAKENHI 16081205 .

\section{References}

[1] J. Jortner, L. Mever, S. A. Rice, and E. G. Wilson, J. Chem. Phys. 42 (1965) 4250.

[2] S. Mihara et al. [MEG Collaboration], J. Phys.: Conf. Ser. 308(2011)012009.

[3] J. Haba, Summary talk at the International Conference on new photon-detectors (PD07), June 27-29, 2007, Kobe, Japan; http://www-conf.kek.jp/PD 07

[4] S. Ritt et al., Nucl. Instr. and Meth. A623 (2010) 486.

[5] H. Otono, et al., PoS (PD07)007, 2007; J. Janicskó Csáthy et al., Nucl. Instr. and Meth. A654 (2011) 225. 